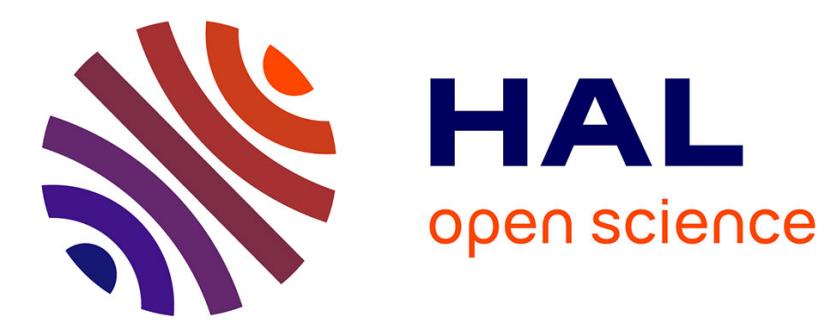

\title{
Trapping and reaction on inhomogeneous structues
}

Davide Cassi

\section{To cite this version:}

Davide Cassi. Trapping and reaction on inhomogeneous structues. Philosophical Magazine, 2010, pp.1. 10.1080/14786435.2010.534743 . hal-00654135

\section{HAL Id: hal-00654135 \\ https://hal.science/hal-00654135}

Submitted on 21 Dec 2011

HAL is a multi-disciplinary open access archive for the deposit and dissemination of scientific research documents, whether they are published or not. The documents may come from teaching and research institutions in France or abroad, or from public or private research centers.
L'archive ouverte pluridisciplinaire HAL, est destinée au dépôt et à la diffusion de documents scientifiques de niveau recherche, publiés ou non, émanant des établissements d'enseignement et de recherche français ou étrangers, des laboratoires publics ou privés. 


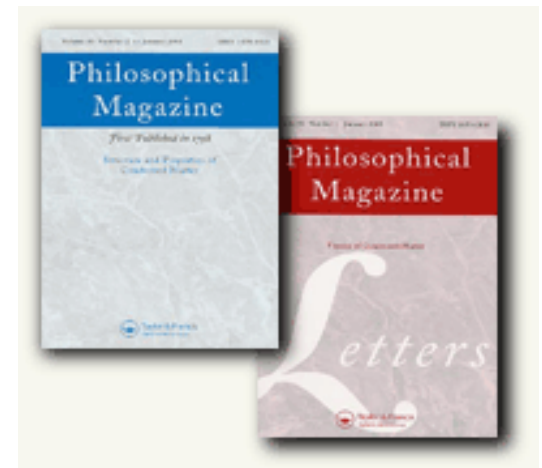

\section{Trapping and reaction on inhomogeneous structues}

\begin{tabular}{|r|l|}
\hline Journal: & Philosophical Magazine \& Philosophical Magazine Letters \\
\hline Manuscript ID: & TPHM-10-May-0182.R1 \\
\hline Journal Selection: & Philosophical Magazine \\
\hline Date Submitted by the \\
Author: & 06-Oct-2010 \\
\hline Complete List of Authors: & Cassi, Davide; Università di Parma, Dipartimento di Fisica \\
\hline Keywords: & diffusion, disorder, amorphous materials \\
\hline Keywords (user supplied): & trapping, reactions, graphs \\
\hline &
\end{tabular}

\section{SCHOLARONE ${ }^{m}$ \\ Manuscripts}




\title{
Trapping and reaction on inhomogeneous structures
}

\author{
Davide Cassi \\ Dipartimento di Fisica, Università di Parma, \\ viale Usberti 7/a, 43100 Parma, ITALY
}

(Date textdate; Received text; Revised text; Accepted text; Published text)

\begin{abstract}
We consider the problem of two chemical species, A and B, undergoing an annihilation process $\mathrm{A}+\mathrm{B} \rightarrow \mathrm{B}$, on generic discrete inhomogeneous structures, such as disordered solids, glasses, fractals, polymer networks and gels. Two particular cases are analysed: in the fist one A is immobile and $\mathrm{B}$ is diffusing (target decay process), in the second one $\mathrm{A}$ is diffusing and B is immobile (trapping process). The survival probability of A is analytically calculated in the limit of large times, showing that, while for the target decay it is related to the spectral dimension $\widetilde{d}$ of the structure, for the trapping problem it depends, in general, on a different anomalous dimension, we call the exploration dimension.

PACS numbers: 82.33.-z, 82.39.Rt, 05.40.-a
\end{abstract}

http://mc.manuscriptcentral.com/pm-pml 


\section{INTRODUCTION}

Diffusion limited reactions are deeply affected by geometry and topology. This phenomenon can be easily evidenced using the random walk model, where the chemical species are described by random walkers diffusing on a given structure $[1,2]$. On regular lattices and on homogeneous structures (i.e. on structures where all sites are topologically equivalent), it is possible to prove simple analytical relations between survival probabilities and basic random walks functions, making explicit the dependence on universal geometrical parameters such as spatial dimension. The case of irregular structures is more complex, due to the absence of symmetries allowing for a reduction of the degrees of freedom involved in analytical calculations. Therefore, in general, it is not possible to relate time decays and survival probabilities to simple geometrical parameters characterizing the underlying structures.

In this paper, we analyze two typical cases of diffusion limited annihilation reactions, where the chemical species $A$ disappears when it meets the chemical species $B: A+B \rightarrow B$.

In the first case, the A species is immobile, and it acts as a target for the moving traps B. This case is known as the target reaction [3].

In the second case the opposite happens: $\mathrm{A}$ is moving and it is trapped when it reaches a site where a species B (trap) is present. This is the case of the trapping reaction [4].

Even if at a first sight the two cases appear rather similar, they cannot be reduced to each other, even on the simplest geometrical structures. Indeed, while the target problem can be analytically solved, on regular lattices and homogeneous structures [3, 5], the trapping problem requires some important mathematical approximations. However, on these particular structures, the asymptotic behaviour of survival probabilities can be expressed in terms of the fundamental quantity $S(t)$, the mean number of distinct sites visited by a random walk after time t, giving rise to similar decay laws.

This is not the case for inhomogeneous structures, where, as we will show in the following, the two asymptotic behaviours can be very different.

\section{BASIC DEFINITIONS}


Let us begin by introducing some basic definitions concerning graphs and random walks $[6]:$

A graph $\mathcal{G}$ is a countable set $V$ of vertices (or sites) $(i)$ connected pairwise by a set $E$ of unoriented links (or bonds) $(i, j)=(j, i)$.

The graph topology can be algebraically represented introducing its adjacency matrix $A_{i j}$ given by:

$$
A_{i j}=\left\{\begin{array}{l}
1 \text { if }(i, j) \in E \\
0 \text { if }(i, j) \notin E
\end{array}\right.
$$

and the coordination number of site $i$, which is the number of nearest neighbors of $i$, is given by $z_{i}=\sum_{j} A_{i j}$.

The discrete time simple random walk on a graph $\mathcal{G}$ is defined by assuming that at each time step $t$ the walker can only jump to a nearest neighbor site, and that all nearest neighbor sites can be reached with the same probability. Therefore, we can define the jumping probabilities $p_{i j}$ between sites $i$ and $j$ by

$$
p_{i j}=\frac{A_{i j}}{z_{i}}=\left(Z^{-1} A\right)_{i j}
$$

where $Z_{i j}=z_{i} \delta_{i j}$.

Now we introduce the functions $P_{i j}(t)$, each representing the probability of being in site $j$ at time $t$ for a walker starting from site $i$ at time 0 , and the first passage probabilities $F_{i j}(t)$, each representing, for $j \neq i$, the conditional probability for a walker starting from $i$ of reaching for the first time the site $j$ in $t$ steps, and, for $i=j$, the probability of returning to the starting point $i$ for the first time after $t$ steps $\left(F_{i i}(0)=0\right)$.

The fundamental relation between the $P_{i j}(t)$ and the $F_{i j}(t)$ is given by

$$
P_{i j}(t)=\sum_{k=0}^{t} F_{i j}(k) P_{j j}(t-k)+\delta_{i j} \delta_{t 0} .
$$

Introducing the generating functions $\tilde{P}_{i j}(\lambda)$ and $\tilde{F}_{i j}(\lambda)$ by the definition

$$
\tilde{f}(\lambda)=\sum_{t=0}^{\infty} \lambda^{t} f(t)
$$

from eq.(3) we obtain the simpler relation

$$
\tilde{P}_{i j}(\lambda)=\tilde{F}_{i j}(\lambda) \tilde{P}_{j j}(\lambda)+\delta_{i j}
$$


which will be useful in the proof of our main results.

On infinite graphs, representing real systems in the thermodynamic limit, $P_{i i}(t)$ vanishes for $t \rightarrow \infty$. If the graph can be embedded in a finite dimensional Euclidean space, and if the coordination numbers are bounded, i.e. if $\exists z_{\max } \mid z_{i} \leq z_{\max } \forall i \in V$, then $P_{i i}(t)$ vanishes typically as a power law, whose exponent allows to define the so called (local) spectral dimension $\widetilde{d}$, which is the natural generalization of the Euclidean dimension for dynamic processes [6]:

$$
P_{i i}(t) \sim p_{i} t^{-\widetilde{d} / 2} \quad \text { for } \quad t \rightarrow \infty \quad \forall i \in \mathcal{G}
$$

with $\widetilde{d} \geq 1$, where $p_{i} \equiv p_{0} z_{i}$ (i.e. it depends only on the coordination number of $i$ ) if $\widetilde{d} \leq 2$. For $\widetilde{d} \leq 2, \tilde{F}_{i j}(1)=1$, i.e., the probability of ever reaching any site starting from any site is 1 , and the graph is called recurrent. For $\widetilde{d}>2, \tilde{F}_{i j}(1)<1$, and the graph is called transient.

\section{THE TARGET DECAY PROBLEM}

Now we can define the target decay problem on an infinite graph, following the formalism introduced in [7].

For more technical details on this subject, see [8], where the problem was first analyzed.

Since each target decays independently of the other ones, we can study the decay of a single target without loss of generality. Let us suppose a target molecule A is placed at site $k$, while, at time $t=0$, the $\mathrm{B}$ molecules are randomly and independently distributed over the other sites, with average site occupation number $q$. The occupation number distribution at each sites turns out to be Poissonian, and the probability $p(n)$ of finding exactly $n \mathrm{~B}$ molecules at a given site is $p(n)=\frac{q^{n} e^{-q}}{n !}$.For $t>0$, the $\mathrm{B}$ molecules are moving randomly and independently according to the jumping probabilities (2), and the target $\mathrm{A}$ is annihilated when it is reached by one of them. Under these hypotheses, it has been shown [7] that the survival probability $\Phi_{k}(t)$ of target $\mathrm{A}$ at time $t$ is given by

$$
\Phi_{k}(t)=e^{-q \Theta_{k}(t)}
$$


where

$$
\Theta_{k}(t)=\sum_{i \neq k} \sum_{\tau=0}^{t} F_{i k}(\tau)
$$

Now, on homogeneous graphs, $F_{i k}(t)=F_{k i}(t)$, and, due to this symmetry, $\Theta_{k}(t)=$ $S(t)-1$, where $S(t)$ is the number of distinct sites visited by a walker after $t$ steps (on homogeneous graphs it is independent of the starting site $k$ ). This result gives rise to the well known results obtained on $d$-dimensional Euclidean lattices, where, for $t \rightarrow \infty$, $\Theta_{k}(t) \sim \sqrt{t}$ for $d=1, \Theta_{k}(t) \sim t / \ln t$ for $d=2$, and $\Theta_{k}(t) \sim t$ for $d \geq 3$ [3].

On inhomogeneous graphs, $F_{i k}(t) \neq F_{k i}(t)$, and the simple relation mentioned above no longer holds, giving rise to a more complex behavior [7].

Let us proceed to the calculation of the asymptotic behavior of $\Theta_{k}(t)$ by introducing its generating function:

$$
\widetilde{\Theta}_{k}(\lambda)=\sum_{t=0}^{\infty} \lambda^{t} \Theta_{k}(t)=\frac{1}{1-\lambda} \sum_{i \neq k} \tilde{F}_{i k}(\lambda)
$$

From $(3)$, for $i \neq k$, we get $[6] \tilde{F}_{i k}(\lambda)=\frac{\tilde{P}_{i k}(\lambda)}{\tilde{P}_{k k}(\lambda)}=\frac{z_{k}}{z_{i}} \frac{\tilde{P}_{k i}(\lambda)}{\tilde{P}_{k k}(\lambda)}$, therefore $\frac{z_{k}}{z_{\max }} \frac{\tilde{P}_{k i}(\lambda)}{\tilde{P}_{k k}(\lambda)} \leq \tilde{F}_{i k}(\lambda) \leq$ $\frac{z_{k}}{z_{\min }} \frac{\tilde{P}_{k i}(\lambda)}{\tilde{P}_{k k}(\lambda)}$ where $z_{\min } \geq 1$ is the minimum coordination number.

Moreover, since $\sum_{i} P_{k i}(t)=1$ for every $t$, we have that $\sum_{i \neq k} \tilde{P}_{k i}(\lambda)=(1-\lambda)^{-1}-\tilde{P}_{k k}(\lambda)$. Therefore

$$
\frac{z_{k}}{z_{\max }}\left(\frac{1}{(1-\lambda)^{2} \tilde{P}_{k k}(\lambda)}-\frac{1}{1-\lambda}\right) \leq \widetilde{\Theta}_{k}(\lambda) \leq \frac{z_{k}}{z_{\min }}\left(\frac{1}{(1-\lambda)^{2} \tilde{P}_{k k}(\lambda)}-\frac{1}{1-\lambda}\right)
$$

Now we can proceed to the singularity analysis of $\widetilde{\Theta}_{k}(\lambda)$ in order to obtain the asymptotic behavior of $\Theta_{k}(t)$ by applying Tauberian theorems [9]. After some mathematics [8], we get the final result

$$
\Theta_{k}(t) \underset{t \rightarrow \infty}{\longrightarrow} \begin{cases}\frac{\sin \left(\pi \frac{\widetilde{d}}{2}\right)}{\bar{z} p_{0} \pi \frac{\widetilde{d}}{2}} t^{\frac{\widetilde{d}}{2}} & \text { for } \widetilde{d}<2 \\ \frac{1}{\bar{z} p_{0}} \frac{t}{\log t} & \text { for } \widetilde{d}=2 \\ \frac{z_{k}}{\bar{z}_{k} \widetilde{P}_{k k}(1)} t & \text { for } \widetilde{d}>2\end{cases}
$$


where $\frac{1}{\bar{z}_{k}} \equiv \lim _{\lambda \rightarrow 1^{-}}(1-\lambda) \sum_{i} \widetilde{P}_{k i}(\lambda) \frac{1}{z_{i}}=\lim _{\lambda \rightarrow 1^{-}} \sum_{i} \widetilde{P}_{k i}(\lambda) \frac{1}{z_{i}} / \sum_{i} \widetilde{P}_{k i}(\lambda)$, with $z_{\min } \leq \bar{z}_{k} \leq z_{\max }$, is the weighted average of the inverse coordination numbers $1 / z_{i}$, with weights $\widetilde{P}_{k i}(\lambda)$, for $\lambda \rightarrow 1^{-}$. Notice that the hypothesis of boundedness of $z_{i}$ could be replaced with the weaker

condition $\bar{z}_{k}<\infty$, leaving our results unchanged. On recurrent graphs, since $\lim _{\lambda \rightarrow 1^{-}} \frac{\tilde{P}_{k i}(\lambda)}{\tilde{P}_{h i}(\lambda)}=1$, uniformly in $i, \bar{z}_{k}$ turns out to be site independent: $\bar{z}_{k}=\bar{z}$.

Notice that, if the graph is homogeneous, we exactly recover the usual asymptotic form of $S(t)$ : this happens not only for Euclidean lattices [3], but also for regular ultrametric spaces [5], and for Bethe lattices, for which the spectral dimension turns out to be infinite [10]. In other cases, such as fractal structures, even if $\Theta_{k}(t)$ is different from $S_{k}(t)$, it turns out to have the same $t$ dependence for $t \rightarrow \infty$.

\section{THE TRAPPING PROBLEM}

The trapping problem is defined considering the trap molecules B immobile, and randomly and independently distributed over the other sites, with average site occupation number $p$, while the target molecule $\mathrm{A}$ is randomly moving on the graph, starting from a randomly chosen point $i$ at time $t=0$.

The survival probability of $\mathrm{A}$ is given by [4]

$$
\Phi(t)=\left\langle(1-p)^{N_{i}(t)-1}\right\rangle
$$

where $N_{i}(t)$ is the number of distinct sites visited in $t$ steps by a walker starting from $i$ in a given walk, and the average is over all possible walks and over all possible starting points $i$.

For small $p$, a first order development can be introduce, known as the Rosentock's approximation [11] :

$$
\Phi(t)=\exp (k-k S(t))
$$

where $k \equiv-\ln (1-p)$ and $S(t) \equiv\left\langle N_{i}(t)\right\rangle$ is the average number of distinct sites visited by a random walker (the average is, as above, also over all possible starting points). 
As one can easily verify, on homogeneous graphs equation (13) coincides with (7) for $k=q$, suggesting a kind of similarity between the two processes.

However, this is not the case for inhomogeneous structures and we are going to show that the asymptotic behaviour of $S(t)$ turns out to be, in general, different from the asymptotic behaviour of $\Theta_{k}(t)$ described in (11).

Now, $S(t)$ can be expressed in terms of $F_{i j}(t)$ by

$$
S(t)=\left\langle 1+\sum_{j} \sum_{\tau=0}^{t} F_{i j}(\tau)\right\rangle
$$

where the average is over all possible starting sites $i$.

Introducing the generating functions and using (5), we get

$$
\langle\widetilde{S}(\lambda)\rangle=\frac{1}{1-\lambda}+\frac{1}{(1-\lambda)^{2}}\left\langle\sum_{j} \tilde{F}_{i j}(\lambda)\right\rangle=\frac{1}{1-\lambda}+\frac{1}{(1-\lambda)^{2}}\left(\left\langle\sum_{j} \frac{\widetilde{P}_{i j}(\lambda)}{\widetilde{P}_{j j}(\lambda)}\right\rangle-1\right)
$$

Now,

$$
\left\langle\sum_{j} \frac{\widetilde{P}_{i j}(\lambda)}{\widetilde{P}_{j j}(\lambda)}\right\rangle=\left\langle\sum_{j} \frac{z_{i}}{z_{j}} \frac{\widetilde{P}_{i j}(\lambda)}{\widetilde{P}_{i i}(\lambda)}\right\rangle
$$

therefore

$$
\frac{z_{\min }}{z_{\max }} \frac{1}{1-\lambda}\left\langle\frac{1}{\widetilde{P}_{i i}(\lambda)}\right\rangle \leq\left\langle\sum_{j} \tilde{F}_{i j}(\lambda)\right\rangle+1 \leq \frac{z_{\max }}{z_{\min }} \frac{1}{1-\lambda}\left\langle\frac{1}{\widetilde{P}_{i i}(\lambda)}\right\rangle
$$

and

$\frac{z_{\min }}{z_{\max }}\left(\frac{1}{(1-\lambda)^{2}}\left\langle\frac{1}{\widetilde{P}_{i i}(\lambda)}\right\rangle-\frac{1}{1-\lambda}\right) \leq\langle\widetilde{S}(\lambda)\rangle \leq \frac{z_{\max }}{z_{\min }}\left(\frac{1}{(1-\lambda)^{2}}\left\langle\frac{1}{\widetilde{P}_{i i}(\lambda)}\right\rangle-\frac{1}{1-\lambda}\right)$

Even if (18) looks very similar to (10), the presence of the average over all sites can give rise to dramatic differences between the asymptotic behaviours of $\Theta_{k}(t)$ and $S(t)$. Indeed, on inhomogeneous lattices, in general we can have 


$$
\operatorname{sing}\left\langle\frac{1}{\widetilde{P}_{i i}(\lambda)}\right\rangle \nsim \operatorname{sing} \frac{1}{\widetilde{P}_{i i}(\lambda)}
$$

since

$$
\operatorname{sing}\left\langle\frac{1}{\widetilde{P}_{i i}(\lambda)}\right\rangle \sim(1-\lambda)^{\left|\frac{D_{E}}{2}-1\right|}
$$

where $D_{E}$ is the exploration dimension of the graph, and is defined according to the singular behaviour of $\left\langle\frac{1}{\widetilde{P}_{i i}(\lambda)}\right\rangle$ by

$$
\operatorname{sing}\left\langle\frac{1}{\widetilde{P}_{i i}(\lambda)}\right\rangle \sim\left\{\begin{array}{l}
(1-\lambda)^{1-\frac{D_{E}}{2}} \text { if } \lim _{\lambda \rightarrow 1^{-}}\left\langle\frac{1}{\widetilde{P}_{i i}(\lambda)}\right\rangle=0 \\
(1-\lambda)^{\frac{D_{E}}{2}-1} \text { if } \lim _{\lambda \rightarrow 1^{-}}\left\langle\frac{1}{\widetilde{P}_{i i}(\lambda)}\right\rangle>0
\end{array}\right.
$$

On lattices $D_{E}=d$, but on inhomogeneous graphs it, can be different from $\tilde{d}[12]$. It can be shown [12] that $D_{E}$ determines the long times asymptotic behaviour of $\left\langle F_{i i}(t)\right\rangle$ according to $\left\langle F_{i i}(t)\right\rangle \sim t^{-\left|\frac{D_{E}}{2}-1\right|-1}$.

Finally, from (19), we get

$$
\langle S(t)\rangle \underset{t \rightarrow \infty}{\sim} \begin{cases}t^{\frac{D_{E}}{2}} & \text { for } D_{E}<2 \\ \frac{t}{\log t} & \text { for } D_{E}=2 \\ t & \text { for } D_{E}>2\end{cases}
$$

which exhibits a different behaviour from (11) whenever $D_{E} \neq \widetilde{d}$ and at least one of the two dimensions is $<2$. 


\section{CONCLUSIONS}

We have shown that the target decay problem and the trapping problem in the Rosentock's approximation, which have the same asymptotic behaviour on homogeneous lattices, can exhibit very different decay laws on inhomogeneous structures, since the former depend on the spectral dimension, while the latter depend on the exploration dimension. However, the decay laws have the same asymptotic behaviour even when $D_{E} \neq \tilde{d}$, provided they are both $>2$, suggesting the recovery of a kind of universality in dimensions higher than 2 , even if it is interesting to notice that almost all experimental determinations of spectral dimension for real systems, up to now have given the result $\tilde{d}<2$.

[1] G. H. Weiss Aspects and Applications of the Random Walks (North-Holland, Amsterdam, 1994).

[2] D. ben-Avraham and S. Havlin, Diffusion and Reactions in Fractals and Disordered Systems (Cambridge University Press, Cambridge, UK, 2004).

[3] A. Blumen, G. Zumofen, and J. Klafter, Phys. Rev. B 30, 5379 (1984).

[4] J. Klafter and A. Blumen, J. Chem. Phys. 80, 875 (1984).

[5] G. Zumofen, A. Blumen, and J. Klafter, J. Chem. Phys. 84, 6679 (1986).

[6] R. Burioni and D. Cassi, J. Phys. A 38, R45 (2005).

[7] F. Jasch and A. Blumen, Phys. Rev. E 63, 041108 (2001).

[8] D. Cassi, Phys. Rev. E 80, 030107(R) (2009)

[9] Ph. Flajolet and R. Sedgewick, Analytic Combinatorics (Cambridge University Press, Cambridge, UK, 2009).

[10] D. Cassi, Europhys. Lett. 9, 627 (1989).

[11] H.B. Rosenstock. Phys. Rev. 187, 1166 (1969).

[12] D. Cassi, to be published. 